\title{
Identification and validation of reference genes for the detection of serum microRNAs by reverse transcription-quantitative polymerase chain reaction in patients with bladder cancer
}

\author{
LISHUI WANG, YIMIN LIU, LUTAO DU, JUAN LI, XIUMEI JIANG, GUIXI ZHENG, AILIN QU, HAIYAN WANG, \\ LILI WANG, XIN ZHANG, HUI LIU, HONGWEI PAN, YONGMEI YANG and CHUANXIN WANG
}

Department of Clinical Laboratory, Qilu Hospital, Shandong University, Jinan, Shandong 250012, P.R. China

Received May 5, 2014; Accepted January 22, 2015

DOI: $10.3892 / \mathrm{mmr} .2015 .3428$

\begin{abstract}
Serum microRNAs (miRNAs) have been proposed as novel non-invasive biomarkers for the early detection of cancer. Reverse transcription-quantitative polymerase chain reaction (RT-qPCR) is the most commonly used method for investigating miRNA expression levels, however, the interpretation of RT-qPCR results depends largely on normalization to an appropriate endogenous control. The present study involved 129 patients with non-muscle-invasive bladder cancer (NMIBC), 121 patients with muscle-invasive bladder cancer (MIBC) and 158 healthy controls. The aim of the present study was to determine the most stable reference genes for the investigations of serum miRNA in bladder cancer (BC). MiSeq sequencing was performed and the expression levels of $10 \mathrm{miRNAs}$ and U6 were then measured using RT-qPCR. Following RT-qPCR, five genes (hsa-miR-193a-5p, hsa-miR-16-5p, U6, hsa-miR-191-5p and hsa-let-7d-3p) were selected for stability analysis using geNorm and NormFinder software. These algorithms identified hsa-miR-193a-5p and hsa-miR-16-5p as the most stably expressed reference genes. The availability of hsa-miR-193a-5p and hsa-miR-16-5p was confirmed in an additional cohort. One-way analysis of variance indicated that no significant differences were present in the expression levels among the three groups. Furthermore, miR-148b-3p was selected as a target miRNA to determine the effect of hsa-miR-193a-5p and hsa-miR-16-5p on miRNA quantification. The combined use of hsa-miR-193a-5p and hsa-miR-16-5p enabled the detection of a significant upregulation of miR-148b-3p in the BC serum. The results of the present study demonstrated that normalization of miRNA data, using a combination of hsa-miR-193a-5p and hsa-miR-16-5p as reference genes, may produce reliable and accurate results for the detection of serum miRNAs in BC.
\end{abstract}

Correspondence to: Mr. Chuanxin Wang, Department of Clinical Laboratory, Qilu Hospital, Shandong University, 107 Wenhua Xi Road, Jinan, Shandong 250012, P.R. China

E-mail: cxwang@sdu.edu.cn

Key words: transcription-quantitative polymerase chain reaction, bladder cancer, microRNAs, reverse reference gene

\section{Introduction}

Bladder cancer (BC) is one of the most frequently occurring types of malignancy worldwide (1). In 2012, 73,510 new cases of $\mathrm{BC}$ were diagnosed in the United States, resulting in mortality of 14,880 individuals (2). In patients with bladder cancer, $\sim 70 \%$ are diagnosed with non-muscle-invasive bladder cancer (NMIBC), which is a type of $\mathrm{BC}$ with a good survival rate, but high rate of recurrence. The remaining 30\% of $\mathrm{BC}$ patients have muscle-invasive bladder cancer (MIBC), which often metastasizes and leads to mortality (3). Furthermore, BC is one of the most expensive types of cancer to treat in the United States, accounting for $\sim 3.7$ billion US dollars (2001 values) in direct costs (4). However, the current treatment strategies do not always provide satisfactory results, particularly for disease extending outside the bladder (5). Since the detection of $\mathrm{BC}$ in its early stages improves the chance of successful treatment, early detection and diagnosis of $\mathrm{BC}$ will significantly improve survival rates. At present, urine cytology screening has been widely adopted for the detection of BC. However, it can produce false-positive results due to the subjective morphological criteria (6). Although alternative diagnostic methods including cystoscopic and histological evaluation of multiple bladder biopsies have been used for diagnosis, these methods are impractical for population screening due to their invasiveness and inconvenience (7). Therefore, the development of non-invasive screening assessment, with high sensitivity and specificity for the early detection of $\mathrm{BC}$, has become a major challenge.

MicroRNAs (miRNAs) are endogenous single-stranded small RNA molecules of 19-24 nucleotides in length, which are transcribed from DNA, but are not translated into proteins $(8,9)$. By binding to the 3'-untranslated region of target mRNAs, miRNAs are able to negatively regulate gene expression at the post-transcriptional level (10). Previous studies have demonstrated that miRNAs are frequently dysregulated in human cancer and exert significant effects on cancer development (11). Previous studies have suggested that serum/plasma miRNAs exist in a cell-free form $(12,13)$ and are resistant to the degradation of RNases $(12,14)$. Reverse transcription-quantitative polymerase chain reaction (RT-qPCR) is an effective technique to examine the gene expression levels of circulating miRNAs, and the interpretation of RT-qPCR results depends largely on 
normalization of the data. At present, the use of reference genes is the most widely used method used for normalization, however, normalization to inappropriate reference genes may lead to misinterpretation, confounding or significant deviation of the data $(15,16)$, thus highlighting the importance of selecting suitable reference genes. In previous studies of circulating miRNAs, reference genes have been selected predominantly on the basis of previous literature or their own experience, and a method of selection remains to be fully established. Therefore, it is necessary to systemically evaluate the suitable reference genes for the investigation of circulating miRNAs in different types of human cancer. In a previous study, the combination of miR-191-5p and U6 was identified as suitable for use as reference genes in the investigation of serum microRNAs in patients with colorectal adenocarcinoma and adenoma (17). However, few studies have been performed concerning the identification of reference genes for RT-qPCR analysis of serum miRNAs in BC.

In the present multiphase, case-control study, candidate reference genes were screened using MiSeq sequencing in the first screening phase. In the second selection phase, RT-qPCR and two different algorithms were used to evaluate their expression stability. In the third validation phase, the selected reference genes were confirmed in an independent cohort. In addition, miR-148b-3p was selected as a target miRNA for further validation.

\section{Materials and methods}

Study population and design. The present study enrolled 250 patients with newly diagnosed BC in the Department of Urologic Surgery, Qilu Hospital, Shandong University (Jinan, China) between 2007 and 2013. A total of 176 patients with BC underwent transurethral resection of bladder tumor, 13 patients with $\mathrm{BC}$ underwent partial excision of $\mathrm{BC}$ and 61 patients with $\mathrm{BC}$ underwent total removal of $\mathrm{BC}$. The tumors of 64 patients were on the left bladder wall, 56 were on the right bladder wall, 25 were on the anterior wall, 29 were on the posterior wall, 19 were in the neck of the bladder, 8 were in the apex of bladder and 49 patients had multiple bladder tumors. The size of tumor specimens ranges from $0.1-4 \mathrm{~cm}^{2}$. During the operation, the patients were administered the following anaesthesia: Intravenous drip of midazolam, $0.1 \mathrm{mg} / \mathrm{kg}$; propofol injection, $1.5 \mathrm{mg} / \mathrm{kg}$; cis-benzenesulfonic acid with atracurium, $0.15 \mathrm{mg} / \mathrm{kg}$; sufentanil citrate injection, $2 \mu \mathrm{g} / \mathrm{kg}$. Prior to surgery, fentanyl $(2 \mu \mathrm{g} / \mathrm{kg})$ was administered to the patients and during the surgery, patients received oral sevoflurane. Histological specimens from all the patients were reviewed to confirm the diagnosis of $\mathrm{BC}$, and the tumors were staged based on the following criteria of the Union for International Cancer Control (Geneva, Switzerland): pTa, noninvasive papillary urothelial carcinoma; pT1, tumor invading into the lamina propria; pT2, tumor invading into muscularis propria; pT3, tumor invading into perivesical soft tissue; pT4, tumor invading into an adjacent organ, including the uterus, vagina, prostate, pelvic wall or abdominal wall (18). A group of 158 healthy participants were selected as controls from a large pool of individuals for general health checks at the Healthy Physical Examination Centre of Qilu Hospital (Jinan, China). All the controls were matched to the patients by age and sex (Tables I and II). The present study was performed with the approval of the ethics committee of
Qilu Hospital, Shandong University (Jinan, China) and written informed consent was obtained from each participant.

A multiphase, case-control study was designed to identify suitable candidate reference genes for normalizing the RT-qPCR data of the serum miRNAs in BC (Fig. 1). In the first phase, MiSeq sequencing was performed to screen candidate reference miRNAs in three pooled serum samples from 10 patients with MIBC (stages pT2-pT4), 10 patients with NMIBC (stages pTa and pT1) and 10 controls. Only miRNAs with a minimum of 50 copies in all three pooled samples, and exhibiting no differential expression among the three pooled samples $(\mathrm{P}>0.05)$ were selected as candidate reference genes. In the second phase, RT-qPCR was performed in additional participants (30 patients with MIBC, 30 patients with NMIBC and 35 controls) in order to examine the expression levels of the selected genes, to further validate the most suitable genes. In the third phase, the selected genes were validated in an independent cohort consisting of 61 patients with MIBC, 63 patients with NMIBC and 67 controls. miR-148b-3p was selected as a target miRNA for further validation in 46 patients with $\mathrm{BC}$ and 46 controls.

Sample preparation. Venous blood samples $(5 \mathrm{ml})$ were collected from 158 controls and 250 patients with $\mathrm{BC}$ who had not undergone surgery, chemotherapy or radiotherapy. Venous blood samples were collected using a single use Vacutainer ${ }^{\circledR}$ blood collection tube (BD Biosciences, Franklin Lakes, NJ, USA). The serum was separated within $2 \mathrm{~h}$ of blood collection by centrifugation at $1,500 \mathrm{x}$ g for $10 \mathrm{~min}$, followed by $15 \mathrm{~min}$ high-speed centrifugation $(13,800 \mathrm{x}$; ; Thermo Electro LED GmbH, Osterode, Germany) to completely remove the cell debris. The serum samples were then stored at $-80^{\circ} \mathrm{C}$ until further analysis.

MiSeq sequencing. Equal $1 \mathrm{ml}$ volumes of the serum from 10 patients with MIBC, 10 patients with NIMBC and 10 sex- and age-matched controls were pooled, respectively. An miRNeasy mini kit (Qiagen, Valencia, CA, USA) was used to extract and purify total RNA, including miRNA, according to the manufacturer's instructions. Briefly, the 3' adaptor was ligated sequentially to the miRNA and then selected using PAGE electrophoresis (adaptors and PAGE from Berry Genomics Co., Ltd., Shanghai, China). Subsequently, the 5' adaptor was ligated sequentially to the miRNA. Thus, a pair of adaptors was ligated to the $3^{\prime}$ and $5^{\prime}$ ends of the miRNA and the ligated miRNA molecules were used as templates for cDNA synthesis. The cDNAs were then amplified to establish a cDNA library. A KAPA SYBR FAST Universal qPCR kit (Kapa Biosystems, Inc., Wilmington, MA, USA) was used to determine the library quality based on the following criteria: i) cDNA concentration $>1 \mathrm{nM}$,ii) no dimer contamination. The sequencing analysis was performed using the purified cDNA with a MiSeq Sequencing system (Illumina, Inc., San Diego, CA, USA), according to the manufacturer's instructions. For the MiSeq sequencing, the final reads of each miRNA were determined by normalization with the total reads of all the miRNAs in the sample. Bioinformatics analysis was performed by searching against the miRBase, version 17.0 (http://www.mirbase.org/) to identify known mature miRNAs.

cDNA synthesis and RT-qPCR analysis. The cDNA of the miRNAs was synthesized using a One Step PrimeScript miRNA 
Table I. Characteristics of patients and controls.

\begin{tabular}{|c|c|c|c|}
\hline Characteristic & Screening phase & Selection phase & Validation phase \\
\hline Controls (n) & 10 & 35 & 67 \\
\hline Age $($ mean $\pm \mathrm{SD})$ & $65.38 \pm 14.31$ & $62.15 \pm 12.18$ & $63.30 \pm 12.54$ \\
\hline \multicolumn{4}{|l|}{ Gender (n) } \\
\hline Male & $6(60.0 \%)$ & $19(54.3 \%)$ & $41(61.2 \%)$ \\
\hline Female & $4(40.0 \%)$ & $16(45.7 \%)$ & $26(38.8 \%)$ \\
\hline NMIBC (n) & 10 & 30 & 63 \\
\hline Age $($ mean $\pm \mathrm{SD})$ & $66.72 \pm 13.28$ & $65.31 \pm 14.23$ & $63.46 \pm 12.66$ \\
\hline \multicolumn{4}{|l|}{ Gender (n) } \\
\hline Male & $6(60.0 \%)$ & $17(56.7 \%)$ & $39(61.9 \%)$ \\
\hline Female & $4(40.0 \%)$ & $13(43.3 \%)$ & $24(38.1 \%)$ \\
\hline \multicolumn{4}{|c|}{ Pathological stage (n) } \\
\hline $\mathrm{pTa}$ & $7(70.0 \%)$ & $22(73.3 \%)$ & $38(60.3 \%)$ \\
\hline pT1 & $3(30.0 \%)$ & $8(26.7 \%)$ & $25(39.7 \%)$ \\
\hline MIBC (n) & 10 & 30 & 61 \\
\hline Age $($ mean \pm SD $)$ & $63.27 \pm 13.29$ & $64.53 \pm 11.36$ & $64.71 \pm 14.65$ \\
\hline \multicolumn{4}{|l|}{ Gender (n) } \\
\hline Male & $7(70.0 \%)$ & $19(63.3 \%)$ & $34(55.7 \%)$ \\
\hline Female & $3(30.0 \%)$ & $11(36.7 \%)$ & $27(44.3 \%)$ \\
\hline \multicolumn{4}{|c|}{ Pathological stage (n) } \\
\hline pT2 & $2(20.0 \%)$ & $5(16.7 \%)$ & $9(14.8 \%)$ \\
\hline pT3 & $5(50.0 \%)$ & $14(46.7 \%)$ & $31(50.8 \%)$ \\
\hline pT4 & $3(30.0 \%)$ & $11(36.7 \%)$ & $21(34.1 \%)$ \\
\hline
\end{tabular}

SD, standard deviation; NMIBC, non-muscle-invasive bladder cancer; MIBC, muscle-invasive bladder cancer.

Table II. Characteristics of patients and controls in the target miRNA validation phase.

\begin{tabular}{lccc}
\hline Characteristic & MIBC & NMIBC & Control \\
\hline Number & 20 & 26 & 46 \\
Age (mean \pm SD) & $67 \pm 13.59$ & $65 \pm 15.21$ & \\
Gender (n) & $14(70.0 \%)$ & $17(65.4 \%)$ & $27(58.7 \%)$ \\
Male & $6(30.0 \%)$ & $9(34.6 \%)$ & $19(41.3 \%)$ \\
Female & & & \\
Pathological stage (n) & N/A & $5(19.2 \%)$ & N/A \\
pTa & N/A & N/A & N/A \\
pT1 & $4(20.0 \%)$ & N/A \\
pT2 & $11(55.0 \%)$ & N/A \\
pT3 & $5(25.0 \%)$ & N/A \\
pT4 & & & \\
\hline
\end{tabular}

MIBC, muscle-invasive bladder cancer; NMIBC, non-muscle-invasive bladder cancer; SD, standard deviation.

cDNA Synthesis kit (Takara Bio Inc., Otsu, Japan). The reaction mixture $(20 \mu \mathrm{l})$ contained $10 \mu \mathrm{l} 2 \mathrm{X}$ miRNA Reaction Buffer mix, $2 \mu 1$ miRNA Primescript RT Enzyme mix and $2 \mu 10.1 \%$ bovine serum albumin, which were provided in the kit, as well as $3 \mu \mathrm{l}$ serum mixed with $3 \mu \mathrm{l}$ serum buffer $(2.5 \%$ Tween 20 , $50 \mathrm{mmol} / \mathrm{l}$ Tris and $1 \mathrm{mmol} / \mathrm{l}$ EDTA; Solarbio Science and
Technology Co., Ltd, Beijing, China) (19). The mixture was subsequently incubated at $37^{\circ} \mathrm{C}$ for $60 \mathrm{~min}$, at $85^{\circ} \mathrm{C}$ for $5 \mathrm{sec}$ and then at $4^{\circ} \mathrm{C}$ for $60 \mathrm{~min}$. Following centrifugation at 9,600 $\mathrm{x}$ g for $10 \mathrm{~min}$ at $4^{\circ} \mathrm{C}$, the cDNA was stored at $-20^{\circ} \mathrm{C}$ until use.

The RT-qPCR reaction for the detection of miRNAs was performed in a final volume of $25 \mu \mathrm{l}$ using an ABI PRISM 


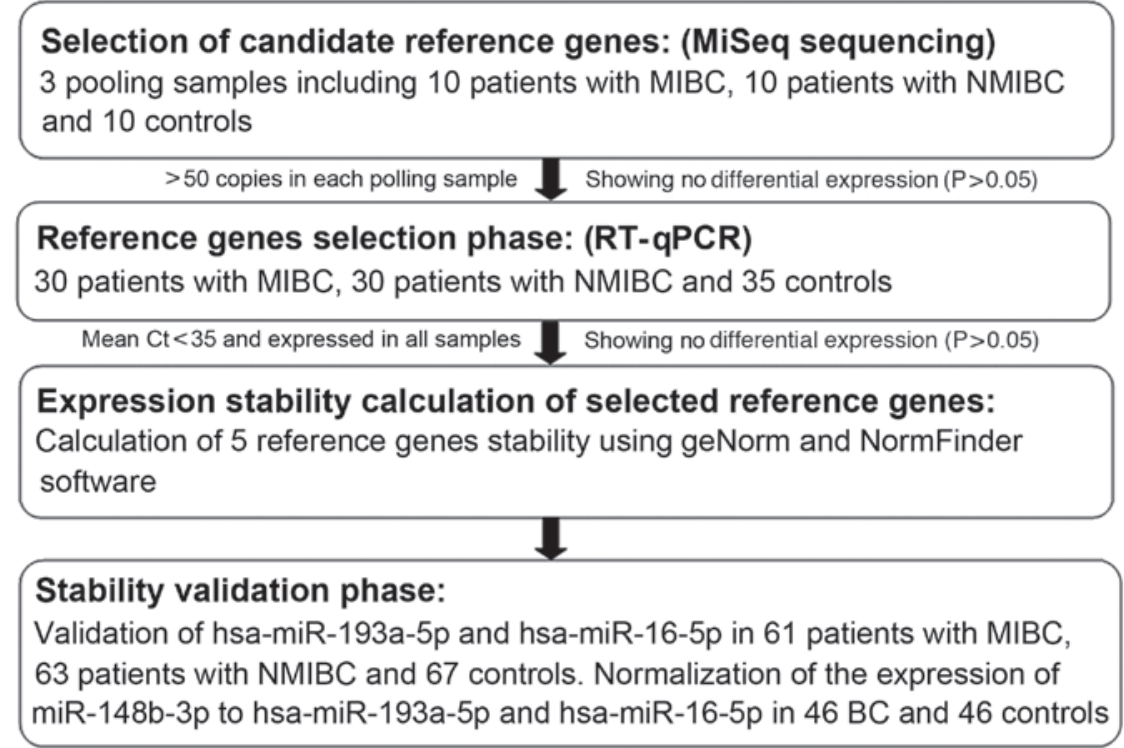

Figure 1. Workflow chart of the design of the present study.

7500 Sequence Detection system (Applied Biosystems Life Technologies, Foster City, CA, USA) using a SYBR PrimeScript miRNA qPCR kit (Takara Bio Inc.). The reaction mixture contained $2.0 \mu 1$ template cDNA, $12.5 \mu$ l SYBR Premix Ex Taq II, $0.5 \mu 1$ DyeII, $2 \mu 15 \mu \mathrm{M}$ forward primer (Ribobio Co., Ltd, Guangzhou, China), $1 \mu 110 \mu \mathrm{M}$ Uni-miR qPCR primer and $7 \mu \mathrm{lddH_{2 }}$ O. The thermal cycling protocol was as follows: An initial $30 \mathrm{sec}$ denaturation step at $95^{\circ} \mathrm{C}$, followed by 45 cycles of $95^{\circ} \mathrm{C}$ for $5 \mathrm{sec}$ and $57^{\circ} \mathrm{C}$ for $34 \mathrm{sec}$. Melting curve analysis was performed at the end of the RT-qPCR run between 65 and $95^{\circ} \mathrm{C}$ to verify the specific amplification. Each measurement was performed in triplicate and the threshold cycle $(\mathrm{Ct})$ value was obtained by the ABI PRISM 7500 software. The candidate reference genes were identified based on the criteria that: i) miRNA was expressed in all samples; ii) the mean $\mathrm{Ct}$ value was $<35$ and iii) the expression of miRNA was not significantly different among the three groups.

Statistical analysis. The average $\mathrm{Ct}$ values were converted into relative quantities for analysis of the overall stability using geNorm and intergroup stability using NormFinder software. One-way analysis of variance was used to calculate the differences in candidate reference genes between the groups. Comparison of the expression levels of miR-148b-3p between different groups was estimated using the Mann-Whitney U test. Statistical analyses and graph construction were performed using SPSS 17.0 (SPSS, Inc., Chicago, IL, USA) and Minitab 15 software (Minitab, Inc., University Park, PA, USA). P $<0.05$ was considered to indicate a statistically significant difference.

\section{Results}

Selection of candidate reference genes. In the MiSeq sequencing results, 206, 259 and 180 miRNAs were detected (>10 copies) in the serum from the MIBC patients, NMIBC patients and controls, respectively. Following normalization of the final reads for each miRNA in the pooled sample of total miRNAs, 23, 16 and 27 miRNAs with $>50$ copies were identified, with no differential expression identified between the MIBC and NMIBC, the MIBC and control or the NMIBC and control groups. According to the selection criteria, described above in 'study population and design', 10 miRNAs were selected as candidate reference genes (Table III).

$R T-q P C R$ analysis of candidate reference genes. In this second phase of the present study, RT-qPCR was performed in an independent cohort to examine the expression levels of 11 genes, including the U6 and 10 selected candidate reference genes. The hsa-miR-10a-5p and hsa-miR-345-5p miRNAs were excluded from further examination as they were not detected in all samples. Based on the $\mathrm{Ct}$ values of the remaining nine genes, no significant differences were observed in the gene expression levels among the three groups $(\mathrm{P}>0.05$; Table IV and Fig. 2). Of the nine candidate reference genes, the $\mathrm{Ct}$ values varied markedly, ranging between 23.19 and 42.97 . The expression levels of four miRNAs (hsa-miR-143-3p, hsa-miR-140-3p, hsa-miR-502-3p and hsa-miR-141-3p) were deemed too low, with median $\mathrm{Ct}$ values $>35$. Therefore, five genes (hsa-miR-193a-5p, hsa-miR-16-5p, U6, hsa-miR-191-5p and hsa-let-7d-3p) were included for subsequent stability analysis.

geNorm and NormFinder analysis. Table $\mathrm{V}$ lists the stability of the five genes analyzed by geNorm and NormFinder (20). geNorm calculates the stability measure (M) of each gene, and lower values indicate the genes with higher stability. Subsequently, geNorm excludes the least stable miRNA following each calculation and recalculates the $\mathrm{M}$ values of the remaining candidate reference genes, with the two most stable miRNAs remaining (Fig. 3). It is also able to calculate the optimal number of reference genes needed for normalization by generating a normalization factor (NF), which is first calculated with the two most stable genes, and then subsequently recalculated in a stepwise-manner by including the most stable remaining gene. The pair-wise variation $\left(\mathrm{V}_{\mathrm{n} / \mathrm{n}+1}\right)$ of two sequential $\mathrm{NFs}\left(\mathrm{NF}_{\mathrm{n}}\right.$ and $\left.\mathrm{NF}_{\mathrm{n}+1}\right)$ is then calculated, with the lowest $\mathrm{V}_{\mathrm{n} / \mathrm{n}+1}$ indicating the optimal number of reference genes. The cut-off value for the lowest $V_{n / n+1}$ is 0.15 , 
Table III. Characteristics of the candidate reference miRNAs.

\begin{tabular}{|c|c|c|c|c|}
\hline miRNA & Mature miRNA sequence $\left(5^{\prime}-3^{\prime}\right)$ & $\begin{array}{l}\text { Copies in } \\
\text { MIBC (n) }\end{array}$ & $\begin{array}{c}\text { Copies in } \\
\text { NMIBC (n) }\end{array}$ & $\begin{array}{l}\text { Copies in } \\
\text { control (n) }\end{array}$ \\
\hline hsa-miR-193a-5p & UGGGUCUUUGCGGGCGAGAUGA & 5911 & 7507 & 5759 \\
\hline hsa-miR-191-5p & CAACGGAAUCCCAAAAGCAGCUG & 2714 & 3320 & 2937 \\
\hline hsa-miR-16-5p & UAGCAGCACGUAAAUAUUGGCG & 1110 & 1422 & 1226 \\
\hline hsa-miR-10a-5p & UACCCUGUAGAUCCGAAUUUGUG & 462 & 559 & 431 \\
\hline hsa-miR-345-5p & GCUGACUCCUAGUCCAGGGCUC & 435 & 412 & 314 \\
\hline hsa-miR-143-3p & UGAGAUGAAGCACUGUAGCUC & 312 & 394 & 272 \\
\hline hsa-miR-140-3p & UACCACAGGGUAGAACCACGG & 192 & 245 & 263 \\
\hline hsa-miR-502-3p & AAUGCACCUGGGCAAGGAUUCA & 121 & 156 & 184 \\
\hline hsa-let-7d-3p & CUAUACGACCUGCUGCCUUUCU & 67 & 72 & 67 \\
\hline hsa-miR-141-3p & UAACACUGUCUGGUAAAGAUGG & 69 & 58 & 55 \\
\hline
\end{tabular}

miRNA, microRNA; MIBC, muscle-invasive bladder cancer; NMIBC, non-muscle-invasive bladder cancer.

Table IV. Ct values of the candidate reference miRNAs.

\begin{tabular}{lccccccc}
\hline & & & & \multicolumn{3}{c}{ Mean Ct \pm SD } \\
\cline { 5 - 7 } Name & Ct Range & Ct min & Ct max & MIBC & NMIBC & Control & P-value \\
\hline U6 & 5.42 & 23.19 & 28.61 & $25.50 \pm 1.03$ & $25.56 \pm 0.79$ & $25.69 \pm 0.94$ & 0.701 \\
hsa-miR-16-5p & 3.25 & 31.03 & 34.28 & $32.65 \pm 0.78$ & $32.43 \pm 0.78$ & $32.50 \pm 0.74$ & 0.544 \\
hsa-miR-191-5p & 5.05 & 29.22 & 34.27 & $31.66 \pm 0.91$ & $31.78 \pm 0.95$ & $31.45 \pm 1.05$ & 0.391 \\
hsa-miR-193a-5p & 2.30 & 30.31 & 32.61 & $31.37 \pm 0.51$ & $31.55 \pm 0.50$ & $31.33 \pm 0.55$ & 0.238 \\
hsa-let-7d-3p & 5.64 & 30.17 & 35.81 & $32.64 \pm 1.18$ & $32.92 \pm 1.14$ & $32.69 \pm 1.12$ & 0.599 \\
hsa-miR-140-3p & 9.25 & 32.07 & 41.32 & $35.21 \pm 1.50$ & $35.69 \pm 1.65$ & $35.39 \pm 1.67$ & 0.508 \\
hsa-miR-143-3p & 10.01 & 31.94 & 41.95 & $35.31 \pm 1.89$ & $35.48 \pm 1.53$ & $34.89 \pm 1.46$ & 0.320 \\
hsa-miR-502-3p & 13.34 & 29.63 & 42.97 & $35.02 \pm 1.84$ & $35.35 \pm 2.17$ & $34.72 \pm 2.53$ & 0.527 \\
hsa-miR-141-3p & 16.04 & 24.99 & 41.03 & $35.14 \pm 2.07$ & $35.36 \pm 2.46$ & $35.37 \pm 2.57$ & 0.916 \\
\hline
\end{tabular}

Ct, threshold cycle; miRNA, microRNA; SD, standard deviation; MIBC, muscle-invasive bladder cancer; NMIBC, non-muscle-invasive bladder cancer.

Table V. Stability ranking of the candidate reference genes and the most stable combination.

\begin{tabular}{|c|c|c|c|c|}
\hline \multirow[b]{2}{*}{ Rank } & \multicolumn{2}{|c|}{ NormFinder } & \multicolumn{2}{|c|}{ geNorm } \\
\hline & Gene & Stability & Gene & Stability (M) \\
\hline 1 (highest) & hsa-miR-193a-5p & 0.088 & hsa-miR-193a-5p & 1.065 \\
\hline 2 & hsa-miR-16-5p & 0.131 & hsa-miR-16-5p & 1.164 \\
\hline 3 & U6 & 0.158 & U6 & 1.223 \\
\hline 4 & hsa-miR-191-5p & 0.169 & hsa-miR-191-5p & 1.271 \\
\hline 5 (lowest) & hsa-let-7d-3p & 0.204 & hsa-let-7d-3p & 1.378 \\
\hline Most stable combination & hsa-miR-193a-5p/16-5p & 0.080 & hsa-miR-193a-5p/16-5p & 0.957 \\
\hline
\end{tabular}

above which the additional gene is necessary for normalization. However, 0.15 is not an absolute cut-off value, and the number of reference genes can be determined according to the requirement of the investigation (21). In the present study, geNorm analysis suggested that all of the five genes (hsa-miR-193a-5p, hsa-miR-16-5p, U6, hsa-miR-191-5p and hsa-let-7d-3p) were suitable as candidate reference genes. NormFinder and geNorm identified hsa-miR-193a-5p as the most stably expressed 


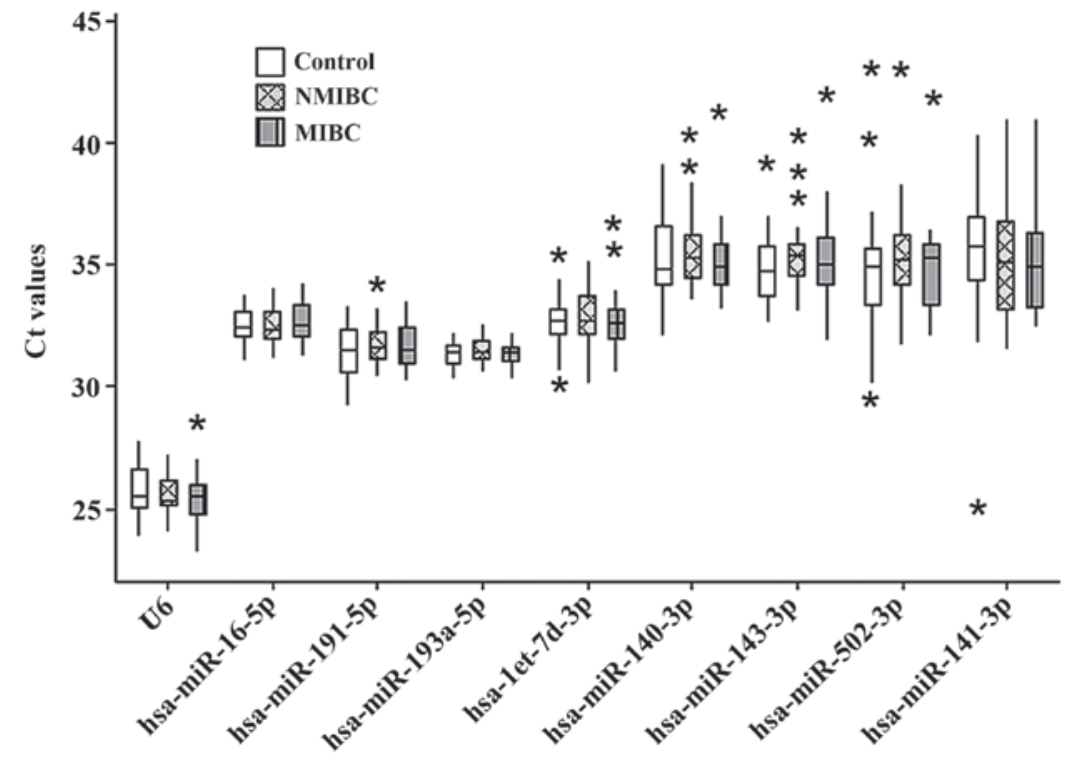

Figure 2. Ct values of the candidate reference genes in the MIBC, NMIBC and control groups. Values are presented as the mean \pm standard deviation and outliers are represented by '*'. No significant differences were observed between the three groups $(\mathrm{P}>0.05)$. Ct, threshold cycle; miR, microRNA; MIBC, muscle-invasive bladder cancer; NMIBC, non-muscle-invasive bladder cancer.

A
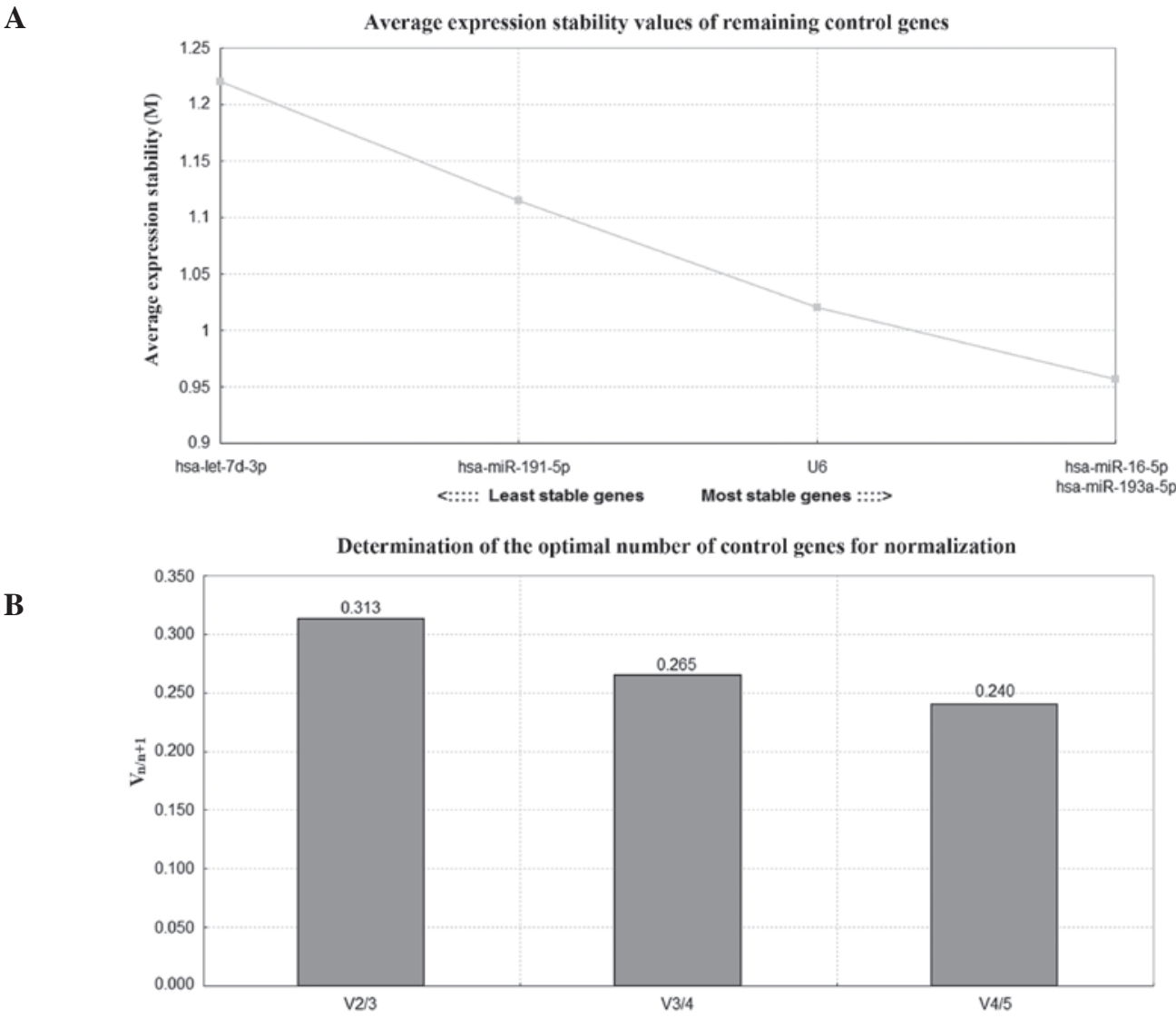

Figure 3. geNorm analysis of the candidate reference genes. (A) Stability ranking of the candidate reference genes. The least stable of the genes with the highest $M$ values was excluded in a stepwise-manner until the two most stable genes remained, identified as hsa-miR-193a-5p and hsa-miR-16-5p. (B) Determination of the optimal reference genes. The $\mathrm{X}$-axis represents the pair-wise variations $\left(\mathrm{V}_{\mathrm{n} / \mathrm{n}+1}\right)$ of NF2 and NF3, NF3 and NF4, NF4 and NF5; the Y-axis indicates the pair-wise variation values $\left(\mathrm{V}_{\mathrm{n} / \mathrm{n}+1}\right)$. The geNorm output file indicated that optimal normalization of gene expression was attainable using the five selected reference genes. miR, microRNA; NF, normalization factor.

reference gene and selected hsa-miR-193a-5p and hsa-miR-16-5p as the pair of reference genes with the highest stability.
Gene validation. The stability of hsa-miR-193a-5p, hsa-miR-16-5p and the combination of hsa-miR-193a-5p and 


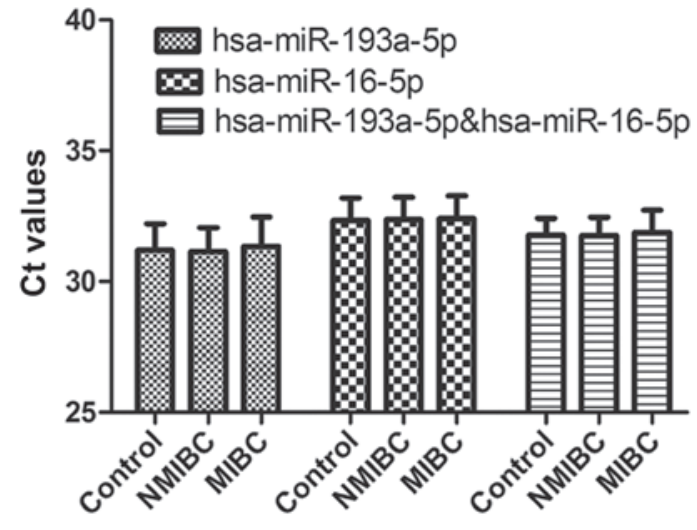

Figure 4. Ct values of hsa-miR-193a-5p, hsa-miR-16-5p and the combination of hsa-miR-193a-5p and hsa-miR-16-5p in the three groups. Values are presented as the mean \pm standard deviation. No significant differences were observed among the three groups $(\mathrm{P}>0.05)$. Ct, threshold cycle; miR, microRNA; MIBC, muscle-invasive bladder cancer; NMIBC, non-muscle-invasive bladder cancer.

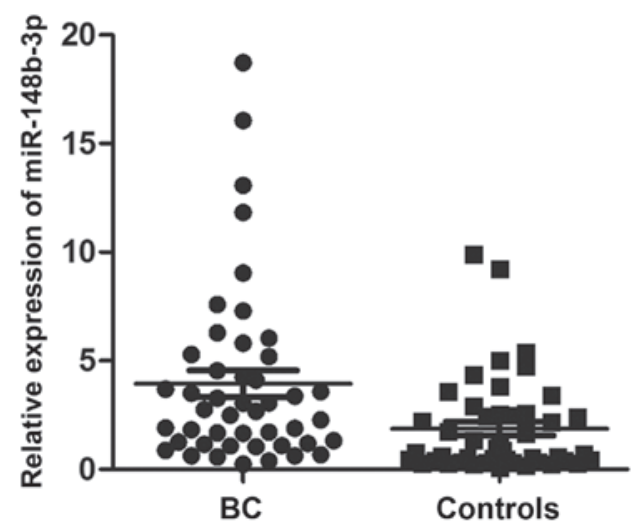

Figure 5. Expression of miR-148b-3p normalized with the combination of hsa-miR-193a-5p and hsa-miR-16-5p. Significant upregulation of miR-148b-3p was detected in the $\mathrm{BC}$ group $(\mathrm{P}<0.05$, compared with the control). miR, microRNA; BC, bladder cancer.

hsa-miR-16-5p were further confirmed with RT-qPCR in an independent cohort (Fig. 4). No significant differences in expression levels were observed among the three groups $(\mathrm{P}>0.05)$. In addition, to determine the effect of the two reference genes on the quantification of miRNAs, circulating miR-148b-3p was selected as the target miRNA, according to the sequencing results, and it has been demonstrated to have potential in evaluating $\mathrm{BC}$ (22). The expression of miR-148b-3p was significantly higher in patients with $\mathrm{BC}$ compared with the controls, using hsa-miR-193a-5p or hsa-miR-16-5p as reference genes $(\mathrm{P}<0.05$; data not shown). This difference remained significant following normalization of the data with the combination of hsa-miR-193a-5p and hsa-miR-16-5p (P<0.05; Fig. 5).

\section{Discussion}

Cell free miRNAs have been reported to be released and circulate in the blood of patients with cancer. Previous studies have also demonstrated that there are distinct miRNA expression patterns associated with specific types of cancer $(23,24)$. The dysregulation of miRNA expression may be an early event during tumorigenesis, thus the detection of differentially expressed circulating miRNAs in easily accessible surrogate tissues, including serum or plasma, may be of value for the early detection of cancer and real-time monitoring of disease progression, including BC (25).

There are currently three methods, including next generation sequencing, chip-based microarrays and RT-qPCR, which are widely used for the identification of miRNA expression patterns $(26,27)$. Although next generation sequencing and chip-based microarrays can be used to simultaneously filter or screen hundreds of miRNAs in a single sample, the results from these high-throughput methods require further validation by RT-qPCR $(28,29)$. In order to obtain reliable RT-qPCR results, a valid standard for normalization is required to minimize technical-associated effects, and remove systematic bias and experimental variation. In previous studies, input volume and spike-in synthetic RNA have been used for normalization of RT-qPCR results $(30,31)$. However, adding the same volume of samples does not ensure an equal quantity of total RNA, while adding spiked-in RNAs only corrects technical variations in miRNA isolation (32). Therefore, selecting appropriate reference genes as endogenous controls has become the most common normalization method for accurate RT-qPCR analysis (33).

It has been demonstrated that inappropriate reference genes can markedly affect overall RT-qPCR results (34). In a previous study of colorectal cancer, the use of inappropriate reference genes resulted in alterations in gene expression, which are biological meaningful (16). In addition, sample type and study design require consideration when selecting a reference gene, as a previous study did not identify any reference genes with constant expression levels in all sample types when exposed to different experimental conditions (21). As a consequence, the expression stability of reference genes requires verification in experiments with different designs $(32,35)$. In previous studies, several miRNAs have been identified as reference genes for the RT-qPCR analysis of serum miRNAs, including miR-484 and miR-191 in breast cancer (36), and miR-16 and miR-93 in gastric cancer (34). To the best of our knowledge, no widely accepted reference genes have been reported for normalizing serum miRNA RT-qPCR data in BC.

U6 is a gene, which is used as a stable reference gene in the investigation of miRNA in different tissues. However, several studies have observed that U6 may be not reliable for the normalization of serum miRNAs $(37,38)$. Therefore, in the present study, U6 was also included for RT-qPCR analysis. Of the 11 genes examined, six miRNAs (hsa-miR-10a-5p, hsa-miR-345-5p, hsa-miR-143-3p, hsa-miR-140-3p, hsa-miR-502-3p and hsa-miR-141-3p) were identified with no or low expression ( $\mathrm{Ct}$ values $>35$ ) and were excluded for evaluation, while the remaining five genes (hsa-miR-193a-5p, hsa-miR-16-5p, U6, hsa-miR-191-5p and hsa-let-7d-3p), which were expressed in all samples and had a mean $\mathrm{Ct}$ value of $<35$, were analyzed using geNorm and NormFinder for stability. geNorm is a pairwise comparison model, which calculates the optimal number of required reference genes to obtain reliable results from RT-qPCR, while NormFinder uses an analysis of variance-based model to estimate intra- and intergroup variations in reference genes (39). In the present study, based on NormFinder and geNorm analysis, hsa-miR-193a-5p was identified as the most suitable gene with constant expression, and the combination of hsa-miR-193a-5p and hsa-miR-16-5p 
was recommended as the most suitable pair of reference genes for serum miRNA investigations in BC. In addition, two methods were used in the validation of hsa-miR-193a-5p and hsa-miR-16-5p, including the analysis of expression levels in a large independent cohort and validation with miR-148b-3p as a target miRNA in an additional cohort. These results were consistent with a previous study, which demonstrating upregulated levels of miR-148b-3p in serum from patients with BC (22).

In conclusion, the results of the present study demonstrated that normalization of miRNAs RT-qPCR data using a combination of hsa-miR-193a-5p and hsa-miR-16-5p as reference genes may produce reliable and accurate results for serum miRNA investigations in BC. However, since the samples size was small, a large sample size and multicenter trials are required to confirm this conclusion.

\section{Acknowledgements}

The authors would like to thank Chengjun Zhou and Junhui Zhen for their assistance in the histological evaluation of samples.

\section{References}

1. Jemal A, Siegel R, Ward E, Hao Y, Xu J and Thun MJ: Cancer statistics, 2009. CA Cancer J Clin 59: 225-249, 2009.

2. Siegel R, Naishadham D and Jemal A: Cancer statistics, 2012. CA Cancer J Clin 62: 10-29, 2012.

3. Kaufman DS, Shipley WU and Feldman AS: Bladder cancer. Lancet 374: 239-249, 2009.

4. Botteman MF, Pashos CL, Redaelli A, Laskin B and Hauser R: The health economics of bladder cancer: A comprehensive review of the published literature. Pharmacoeconomics 21: $1315-1330,2003$

5. Malmström PU: Why has the survival of patients with bladder cancer not improved? BJU Int 101: 267-269, 2008.

6. Falebita OA, Sweeney P and Lee G: Urine cytology in the evaluation of urological malignancy revisited: is it still necessary. Urol Int 84: 45-49, 2010.

7. Mengual L, Lozano JJ, Ingelmo-Torres M, Gazquez C, Ribal MJ and Alcaraz A: Using microRNA profiling in urine samples to develop a non-invasive test for bladder cancer. Int J Cancer 133 2631-2641, 2013

8. Bartel DP: MicroRNAs: genomics, biogenesis, mechanism, and function. Cell 116: 281-297, 2004

9. Ambros V: The functions of animal microRNAs. Nature 431: 350-355, 2004.

10. Bartel DP: MicroRNAs: Target recognition and regulatory functions. Cell 136: 215-233, 2009.

11. Heneghan HM, Miller N and Kerin MJ: MiRNAs as biomarkers and therapeutic targets in cancer. Curr Opin Pharmacol 10: 543-550, 2010

12. Mitchell PS, Parkin RK, Kroh EM, et al: Circulating microRNAs as stable blood-based markers for cancer detection. Proc Natl Acad Sci USA 105: 10513-10518, 2008.

13. Hunter MP, Ismail N, Zhang X, et al: Detection of microRNA expression in human peripheral blood microvesicles. PLoS ONE 3: e3694, 2008.

14. Chim SS, Shing TK, Hung EC, Leung TY, Lau TK, Chiu RW and Lo YM: Detection and characterization of placental microRNAs in maternal plasma. Clin Chem 54: 482-490, 2008.

15. Genovesi LA, Anderson D, Carter KW, Giles KM and Dallas PB: Identification of suitable endogenous control genes for microRNA expression profiling of childhood medulloblastoma and human neural stem cells. BMC Res Notes 5: 507, 2012.

16. Chang KH, Mestdagh P, Vandesompele J, Kerin MJ and Miller N: MicroRNA expression profiling to identify and validate reference genes for relative quantification in colorectal cancer. BMC Cancer 10: 173, 2010.

17. Zheng G, Wang H, Zhang X, et al: Identification and validation of reference genes for qPCR detection of serum microRNAs in colorectal adenocarcinoma patients. PLoS ONE 8: e83025, 2013
18. Cheng L, Montironi R, Davidson DD and Lopez-Beltran A: Staging and reporting of urothelial carcinoma of the urinary bladder. Mod Pathol 22 (Suppl 2): S70-S95, 2009.

19. Asaga S, Kuo C, Nguyen T, Terpenning M, Giuliano AE and Hoon DS: Direct serum assay for microRNA-21 concentrations in early and advanced breast cancer. Clin Chem 57: 84-91, 2011.

20. Zheng G, Wang H, Zhang X, Yang Y, Wang L, Du L, Li W, Li J, Qu A, Liu Y and Wang C: Identification and validation of reference genes for qPCR detection of serum microRNAs in colorectal adenocarcinoma patients. PLoS One 8: e83025, 2013.

21. Vandesompele J, De Preter K, Pattyn F, Poppe B, Van Roy N, De Paepe A and Speleman F. Accurate normalization of real-time quantitative RT-PCR data by geometric averaging of multiple internal control genes. Genome Biol 3: research0034, 2002.

22. Adam L, Wszolek MF, Liu CG, Jing W, Diao L, Zien A, Zhang JD, Jackson D and Dinney CP: Plasma microRNA profiles for bladder cancer detection. Urol Oncol 31: 1701-1708, 2013.

23. Selth LA, Townley SL, Bert AG, Stricker PD, Sutherland PD, Horvath LG, Goodall GJ, Butler LM and Tilley WD: Circulating microRNAs predict biochemical recurrence in prostate cancer patients. Br J Cancer 109: 641-650, 2013.

24. Köberle V, Kronenberger B, Pleli T, et al: Serum microRNA-1 and microRNA-122 are prognostic markers in patients with hepatocellular carcinoma. Eur J Cancer 49: 3442-3449, 2013.

25. Huang X, Liang M, Dittmar R and Wang L: Extracellular microRNAs in urologic malignancies: Chances and challenges. Int J Mol Sci 14: 14785-14799, 2013.

26. Liu R, Zhang C, Hu Z, et al: A five-microRNA signature identified from genome-wide serum microRNA expression profiling serves as a finger-print for gastric cancer diagnosis. Eur J Cancer 47: 784-791, 2010.

27. Bediaga NG, Davies MP, Acha-Sagredo A, et al: A microRNA-based prediction algorithm for diagnosis of non-small lung cell carcinoma in minimal biopsy material. Br J Cancer 109: 2404-2411, 2013.

28. Bargaje R, Hariharan M, Scaria V and Pillai B: Consensus miRNA expression profiles derived from interplatform normalization of microarray data. RNA 16: 16-25, 2010.

29. Chen X, Ba Y, Ma L, et al: Characterization of microRNAs in serum: A novel class of biomarkers for diagnosis of cancer and other diseases. Cell Res 18: 997-1006, 2008.

30. Pritchard CC, Kroh E, Wood B, Arroyo JD, Dougherty KJ, Miyaji MM, Tait JF and Tewari M: Blood cell origin of circulating microRNAs: A cautionary note for cancer biomarker studies. Cancer Prev Res (Phila) 5: 492-497, 2012.

31. Scheffer AR, Holdenrieder S, Kristiansen G, von Ruecker A, Müller SC, Ellinger J. Circulating microRNAs in serum: novel biomarkers for patients with bladder cancer? World J Urol 32: 353-358, 2014.

32. Zhu HT, Dong QZ, Wang G, Zhou HJ, Ren N, Jia HL, Ye QH and Qin LX: Identification of suitable reference genes for qRT-PCR analysis of circulating microRNAs in hepatitis B virus-infected patients. Mol Biotechnol 50: 49-56, 2012.

33. Lardizábal MN, Nocito AL, Daniele SM, Ornella LA, Palatnik JF and Veggi LM: Reference genes for real-time PCR quantification of microRNAs and messenger RNAs in rat models of hepatotoxicity. PLoS ONE 7: e36323, 2012.

34. Song J, Bai Z, Han W, Zhang J, Meng H, Bi J, Ma X, Han S and Zhang Z: Identification of suitable reference genes for qPCR analysis of serum microRNA in gastric cancer patients. Dig Dis Sci 57: 897-904, 2012.

35. Kreth S, Heyn J, Grau S, Kretzschmar HA, Egensperger R and Kreth FW: Identification of valid endogenous control genes for determining gene expression in human glioma. Neuro-oncol 12: $570-579,2010$.

36. Hu Z, Dong J, Wang LE, et al: Serum microRNA profiling and breast cancer risk: The use of miR-484/191 as endogenous controls. Carcinogenesis 33: 828-834, 2012.

37. Peltier HJ and Latham GJ: Normalization of microRNA expression levels in quantitative RT-PCR assays: Identification of suitable reference RNA targets in normal and cancerous human solid tissues. RNA 14: 844-852, 2008.

38. Wang Y, Tang N, Hui T, Wang S, Zeng X, Li H and Ma J. Identification of endogenous reference genes for RT-qPCR analysis of plasma microRNAs levels in rats with acetaminophen-induced hepatotoxicity. J Appl Toxicol 33: 1330-1336, 2013.

39. Andersen CL, Jensen JL and Ørntoft TF: Normalization of real-time quantitative reverse transcription-PCR data: A model-based variance estimation approach to identify genes suited for normalization, applied to bladder and colon cancer data sets. Cancer Res 64: 5245-5250, 2004. 